\title{
A Non-Relativistic Explanation of the Sagnac Effect
}

\author{
Olivier Serret \\ ESIM Engineer, Cugnaux, France \\ Email: o.serret@free.fr
}

How to cite this paper: Serret, O. (2019) A Non-Relativistic Explanation of the Sagnac Effect. Journal of Modern Physics, 10, 500-514.

https://doi.org/10.4236/jmp.2019.105035

Received: February 25, 2019

Accepted: April 15, 2019

Published: April 18, 2019

Copyright (C 2019 by author(s) and Scientific Research Publishing Inc. This work is licensed under the Creative Commons Attribution International License (CC BY 4.0).

http://creativecommons.org/licenses/by/4.0/

\begin{abstract}
More than a century ago, M. Sagnac realized an experiment with light rays, whose results according to him invalidated the theory of Relativity and validated the hypothesis of the Aether. This hypothesis of the ether was not retained by the scientific community, and relativistic explanations were given to the results of the Sagnac experiment. But these relativistic explanations remain open to criticism because they are various and because the prediction may differ depending on whether the observer's frame of reference is at rest or in motion. The neo-Newtonian mechanics proposes a new explanation which leads to demonstrate the formula $\delta L=-\frac{P \cdot R \cdot \Omega}{c}$ with $P$ the light path. In order to verify this new interpretation, it is proposed to do again the Sagnac experiment with a slightly different light path, a square-shaped path. The results should be at least $30 \%$ lower than those predicted by the theory of Relativity. In which case, the relativistic explanation would be questioned.
\end{abstract}

\section{Keywords}

Sagnac Effect, Relativity Theory, Aether, Ether, Neo-Newtonian Mechanics, Light Path, Gyrolaser

\section{Introduction}

In 1913 Georges Sagnac performed an experiment (bearing his name) [1]. This experiment consists of highlighting the shift of the central fringe as a function of the speed of rotation of the disc. Today its main application is the gyrolaser, used in aeronautics, launchers and space. For G. Sagnac, his experience proved the existence of aether. Nevertheless, this hypothesis of the aether has been supplanted for a century by the theory of Relativity, but the given relativistic explanations are arduous and disputed by some. The purpose of this article is to show 
the paradoxes of the relativistic explanations and then to propose another explanation, that one based on the neo-Newtonian mechanics (which is nothing but the Newtonian mechanics without the principle of equivalence). This article does not deal with optical fiber gyros which fall under a slightly different principle (the optical fiber moves with the disk).

\section{Georges Sagnac's Experiment and Measurement}

\subsection{Georges Sagnac's Experiment}

The rotating interferometer comprises the light source and the receiver, a photographic plate that records the interference fringes located at the focus of a telescope. When the light is sent, from a transmitter placed on the rotating disk, in two opposite directions, the center of interference (which corresponds to the median-band at maximum intensity) shifts as a function of the speed of the disk rotation. This measurement is carried out thanks to the photographic receiver installed on the rotating disc. A description of Sagnac experiment is given in Appendix A.

\subsection{Measurement}

- The interferential displacement " $Z$ " (from one direction to the opposite direction) is measured to be worth:

$$
z=\frac{16 \pi N S}{\lambda c}[\mathrm{~m} / \mathrm{m}]
$$

with values of Table 1.

Numerical application: $z=0.07$. It is the offset of the fringe into two opposite directions.

- Unlike what is generally practiced today, G. Sagnac defines the displacement of the central fringe between a rotation direction and the opposite direction. By reducing the formula with the fringe shift $F S$ (from one direction compared to the state at rest) which is half of the interferential displacement, we get the following formula (see APPENDIX A)

$$
F S=4 \frac{S \Omega}{\lambda c}[\mathrm{~m} / \mathrm{m}]
$$

with $\Omega$, speed of rotation, equal to $22 \pi \mathrm{rad} / \mathrm{s}$.

Table 1. Sagnac values.

\begin{tabular}{cccc}
\hline Symbol & Designation & Value & Unit \\
\hline$N$ & $\begin{array}{c}\text { Number of revolutions } \\
\text { per second } \\
\text { Surface swept by } \\
\text { the light beam }\end{array}$ & 2 & $\mathrm{rps}$ \\
$S$ & Wave length & 0.0866 & $\mathrm{~m}^{2}$ \\
$\lambda$ & Light celerity & 436 & $\mathrm{~nm}$ \\
$\mathcal{C}$ & & $3 \times 10^{8}$ & $\mathrm{~m} / \mathrm{s}$ \\
\hline
\end{tabular}


Numerical application: $F S=0.033$. This is the shift of the central fringe with respect to the center. For example, if the projected interferance $\Lambda=0.5 \mathrm{~mm}$, the Fringe Shift of the center line is $17 \mu \mathrm{m}$.

Note: In his article, at the end of paragraph 2 on the optical vortex effect 1, for $N=2$, G. Sagnac gives as value of the interfrange a value $\Lambda$ "of $0.5 \mathrm{~mm}$ to $1 \mathrm{~mm}$ ", that is to say from simple to double! What is the precision of Sagnac measurement? Does it mean something else?

- To overcome this uncertainty on interfranges, in this paper we will use the "path difference" $\delta L[\mathrm{~m}]$ defined as

$$
\delta L=\lambda \cdot F S
$$

In Sagnac experiment, $\delta L=15 \mathrm{~nm}$.

\section{Usual Measurement Interpretations}

\subsection{According to Newtonian Mechanics}

Newtonian mechanics is not able to correctly describe this experiment. In which case, the two light beams would meet at the center of the photographic receiver, there would be no movement of the central fringe. Newtonian mechanics cannot explain the Fringe Shift.

\subsection{According to Aether Hypothesis}

Hyppolyte Fizeau wanted to prove the existence of the aether with the experience of moving water. In the same way, Georges Sagnac wanted to prove the existence of the aether with his experience. The results are consistent with this hypothesis [2] [3] [4]. The trouble is aether is unable to simply explain the negative result of the Michelson and Morley experiment. Anyway, the aether hypothesis has been supplanted by the theory of Relativity which can give explanation to many astrophysical observations.

\subsection{According to Relativity Theory}

According to the theory of Relativity, the speed of light in the vacuum is a constant whatever the speed of the reference frame is. For Georges Sagnac, first goal of his experience was to invalidate the theory of relativity that could not explain these results with a constant light celerity in both frames. Indeed with the Sagnac experiment, depending on whether one places the observator in the terrestrial reference frame or in the frame of the rotating disk, the meeting of the two opposite light rays would not occur in the same place (see Figure 1). This inconsistency alone should have invalidated the theory of Relativity.

Yet the partisans of Relativity consider that the Sagnac experiment would be on the contrary a new proof of the theory of Relativity. Paul Langevin was the first to provide an explanation involving a "temporal" shift derivating the formula

$$
\delta L(\text { Relativity })=-4 \frac{S \cdot \Omega}{c}
$$




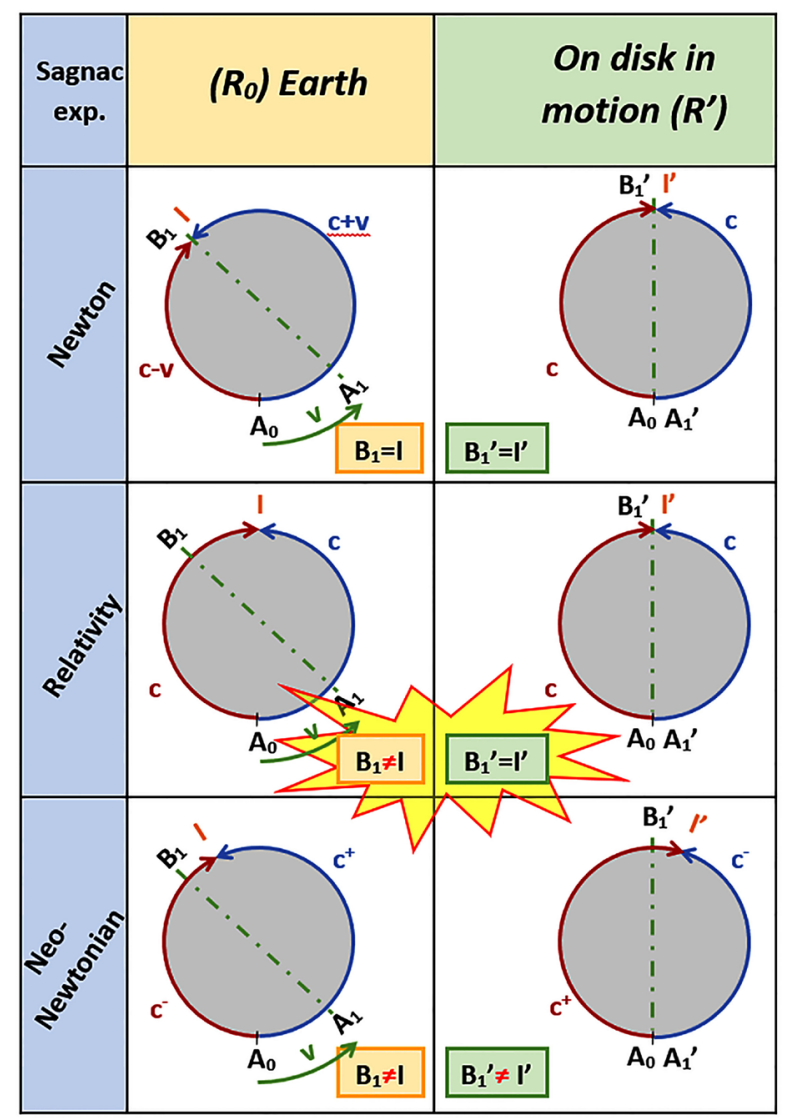

Figure 1. Comparison between different hypotheses.

Since then, other relativistic explanations and derivations have been provided (see APPENDIX B). To give various interpretations for the same phenomenon appears unclear and even suspicious.

\subsection{A Strange Coincidence}

- In the experiment carried out by G. Sagnac, the ratio between the surface $S$ and the perimeter $P$ of this particular polygon is equal to:

$$
4 S \approx P \cdot R
$$

with $R$ radius of the disc.

- In gyroscopic apparatus used in aerospace [5] [6] with an equilateral triangle, the ratio between the surface $S$ and the perimeter $P$ of the triangle is also equal to: (see Figure 2)

$$
4 S=P \cdot R
$$

Nota: in the triangular gyrolaser manufactured by companies, the light beam passes through a resonator (cavity containing an active medium) and that the signal is amplified electronically, filtered, converted into frequency and calibrated. If an indirect link is maintained between the rotational speed and the modification of the signal, the direct link between the speed of rotation and the displacement in meters of the central fringe is masked. 


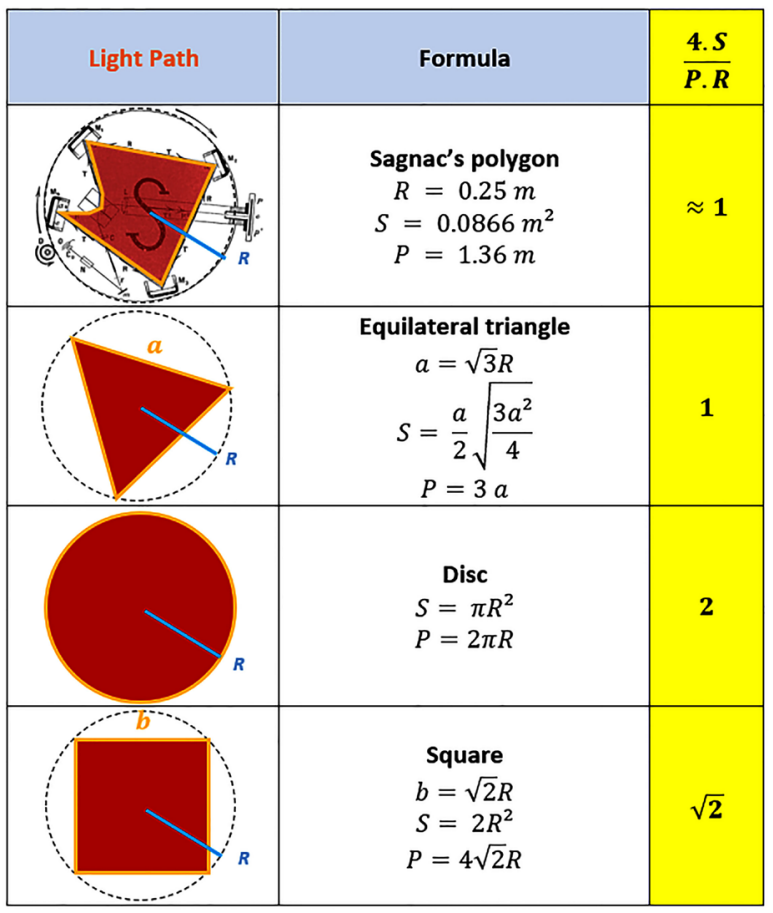

Figure 2. Comparison between Surface and Area.

\section{Neo-Newtonian Interpretation}

\subsection{Neo Newtonian Mechanics}

Neo-Newtonian mechanics is similar to Newtonian mechanics except for very high speed:

- The speed of light " $c$ " is practically equal to the asymptotic limit " $s$ ". So, $c+v$ $\approx c$.

- The addition or the subtraction of the velocities is done according to the conservation of the momentum. So " $c-v=c-(x * v)$ " with $x<1$. In the case of the light beam, $x$ is close to zero.

- For a synthetic approach to neo-Newtonian mechanics see the Presentation paper [7], and for a detailed approach the Related Articles hereafter.

\subsection{Neo Newtonian Explication}

- It is strange that the Fringe Shift depends on the surface of the figure rather than the path traveled by the light. Thus, neo-Newtonian mechanics proposes, and demonstrates (see Appendix C) that the Fringe Shift depends on the path traveled.

The proposed formula is:

$$
\delta L(\text { Neo Newtonian })=-\frac{P \cdot R \cdot \Omega}{c}
$$

- Note that the relativistic derivations are made from the surface of a disk. It is interesting to note that in the case of a circular path, the two approaches give clearly different results (see Appendix C): 


$$
\delta L(\text { Disc } / \text { Neo Newtonian })=\frac{\delta L(\text { Disc } / \text { Relativity })}{2}
$$

This could be a way to discreminate both approaches. Unfortunately, it is not possible to make the light rays travel in a perfectly circular path with mirrors. Let us remember that this article does not deal with the drop-down optical fiber gyrometers where the fiber is rotated with the disk.

\section{Proposal of Experiment}

- Only experiment can help to decide between two interpretations.

It is not possible to simply carry out a real "circular" circulation of the light beams in the vacuum with an infinite number of mirrors. It is here proposed to experiment in a square trajectory (see Figure 3) without resonator nor laser exciter.

- At present time it is difficult to say whether the proponents of Relativity will apply a corrective factor to the square circulation or not. That is why two formulas have been given in the relativistic framework (one by taking up again the formula of the disc, the other by adding a corrective factor proportional to the geometric surface of the square).

- According to Neo-Newtonian, prediction will be done with the single Equation (6).

- Results are in Table 2:

In either case with the square, there is a difference of $(\sqrt{2} / 2=) 30 \%$ to $(\sqrt{2} / \pi=) 55 \%$ to differentiate the predictions of these two theories. This difference from $30 \%$ to $55 \%$ is significant and so it would make possible to discriminate between neo-Newtonian and Relativistic predictions.

\section{Conclusions}

If the Sagnac effect is recognized and today used industrially in gyrolasers for aeronautics and space, its theoretical interpretation remains controversial. For

Table 2. Comparison table between Relativity and Neo-Newtonian predictions.

\begin{tabular}{ccc}
\hline & Relativity & Neo-Newtonian \\
\hline General Formula & $\delta L=-4 \frac{S \cdot \Omega}{c}$ & $\delta L=-\frac{P \cdot R \cdot \Omega}{c}$ \\
Square & $\begin{array}{c}A(\text { disc })=\pi R^{2} \\
S(\text { corrected })=2 R^{2}\end{array}$ & $P=4 \sqrt{2} R$ \\
Formula for square & $\delta L=-4 \frac{\pi R^{2} \cdot \Omega}{c}$ & \\
Example & $\delta L($ corrected $)=-4 \frac{2 R^{2} \cdot \Omega}{c}$ & \\
$R=0.25 \mathrm{~m}$ & $\delta L=-4 \frac{\sqrt{2} \cdot R^{2} \cdot \Omega}{c}$ \\
$\Omega=2 \times 2 \pi \mathrm{rd} / \mathrm{s}$ & $\delta($ corrected $)=21 \mathrm{~nm}$ & $\delta L=15 \mathrm{~nm}$ \\
&
\end{tabular}




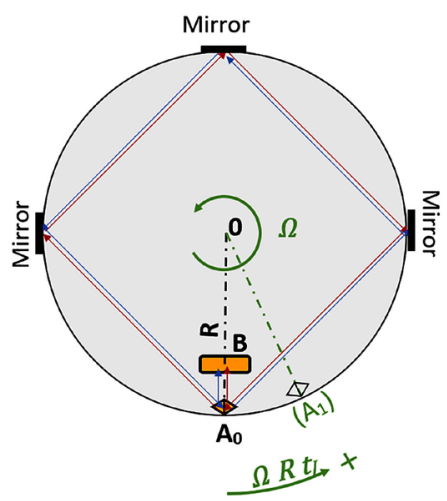

Figure 3. proposed experiment.

the majority of scientists, this would demonstrate the validity of the theory of Relativity, but for some others it invalidates the theory of Relativity and opens the door to other theories such as that of the aether. This was the opinion of Georges Sagnac, the instigator of this experience that bears his name. In this essay, after recalling how neither Newtonian mechanics nor relativistic theory can explain correctly the Sagnac effect, we propose an alternative explanation based on Neo-Newtonian Mechanics (NNM). The Fringe Shift or the difference of light path would not be proportional to the surface swept $S$ but to the light path traveled P. Then we get the formula:

$$
\delta L(\text { Neo-Newtonian })=-\frac{P \cdot R \cdot \Omega}{c} .
$$

Neo-Newtonian Mechanics predicts, except in special cases such as the Sagnac's polygon or the equilateral triangle, a different shift from that predicted by the theory of Relativity. For a perfect square path and without resonator nor exciter, it should show a $30 \%$ to $55 \%$ decrease in the offset. This proposal of experiment, quite similar at first sight to what has already been achieved but nevertheless different, remains to be carried out by a laboratory to discrimeninate both theories.

\section{Conflicts of Interest}

The author declares no conflicts of interest regarding the publication of this paper.

\section{References}

[1] Sagnac, G. (1914) J. Phys. Theor. Appl., 4, 177-195. https://hal.archives-ouvertes.fr/jpa-00241884/document

[2] Su, C.-C. (2000) A Local-Ether Model of Wave Propagation Complying with the Sagnac Correction in the Global Positioning System. IEEE Antennas and Propagation Society International Symposium. Transmitting Waves of Progress to the Next Millennium. 2000 Digest. Held in Conjunction with: USNCIURSI National Radio Science Meeting (C), Salt Lake City, UT, 16-21 July 2000, 1570-1573. https://ieeexplore.ieee.org/abstract/document/874526

[3] Silvertooth, E.W. (1989) Motion through the Ether. EWW. 
http://www.naturalphilosophy.org/pdf/abstracts/abstracts_1248.pdf

[4] Marett, D. (2010) The Premise for Comparing Ether Theories to Relativity Theory. http://www.conspiracyoflight.com/Premise_Ether/Premise_Ether.html

[5] Daussy, C. (2015) Le Gyrolaser. SAGEM.

http://paristech.institutoptique.fr/site.php?id=1010\&fileid $=14007$

[6] Bretenaker, F. (2007) Le Gyrolaser. CNRS.

http://sfp.in2p3.fr/expo/Conf2010/Lasers/Bretenaker.pdf

[7] Serret, O. (2018) General Science Journal, 4.

http://www.gsjournal.net/Science-Journals/Research\%20Papers-Relativity\%20Theor y/Download/7499

[8] Rizzi G. and Ruggiero, M.L. (2004) Relativity in Rotating Frames: Relativistic Physics in Rotating Reference Frames. Springer Science, Berlin.

https://www.springer.com/la/book/9781402018053

[9] Pascoli, G. (2017) Comptes Rendus Physique, 18, 563-569.

https://doi.org/10.1016/j.crhy.2017.10.010

[10] Bouyer, P. (2013) Gyroscopy and Navigation, 5, 20-26.

https://link.springer.com/article/10.1134\%2FS2075108714010039

[11] Arnoux, C. (1994) Gyrolasers-L'effet Sagnac. http://www.cax.free.fr/sagnac/sagnac.html

[12] Malykin, G. (2000) Physics-Uspekhi, 43. https://iopscience.iop.org/article/10.1070/PU2000v043n12ABEH000830/pdf

[13] Spagnou, P. (2013) Sagnac et l'expérience à contresens. Bibnum. https://journals.openedition.org/bibnum/737\#tocto1n4 
RELATED ARTICLES about Neo-Newtonian Mechanics (NNM) CRITICISMS OF THE THEORY OF THE RELATIVITY

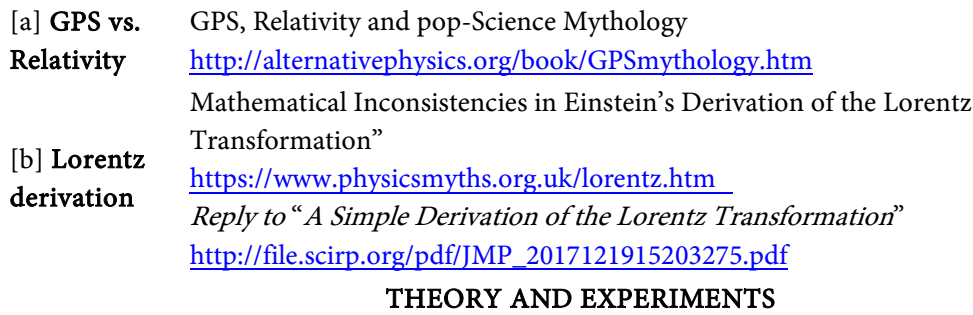

[c] Lorentz How to Demonstrate the Lorentz Factor. Variable Time vs. Variable Inertial Mass factor http://file.scirp.org/pdf/JMP_2015022510573131.pdf Velocity Addition Demonstrated from the Conservation of Linear Momenta, an Alternative Expression

[d] Velocity http://file.scirp.org/pdf/JMP_2015050609513342.pdf

Addition An improvement of the accuracy of Fizeau's experiment http://gsjournal.net/Science-Journals/Research\%20Papers-Relativity\%20Theory/Do wnload/7247 [e] Force in Net Force $F=\gamma^{3}$ ma at High Velocity

the

Synchrotron http://file.scirp.org/pdf/JMP_2016042814580505.pdf

[f] Muons Muon Lifetime would depend of its Energy

Lifetime $\quad$ http://www.mrelativity.net/Papers/51/Muons\%20Serret\%20Millennium.pdf

[g] Sagnac Present article
effect

COSMIC OBSERVATIONS

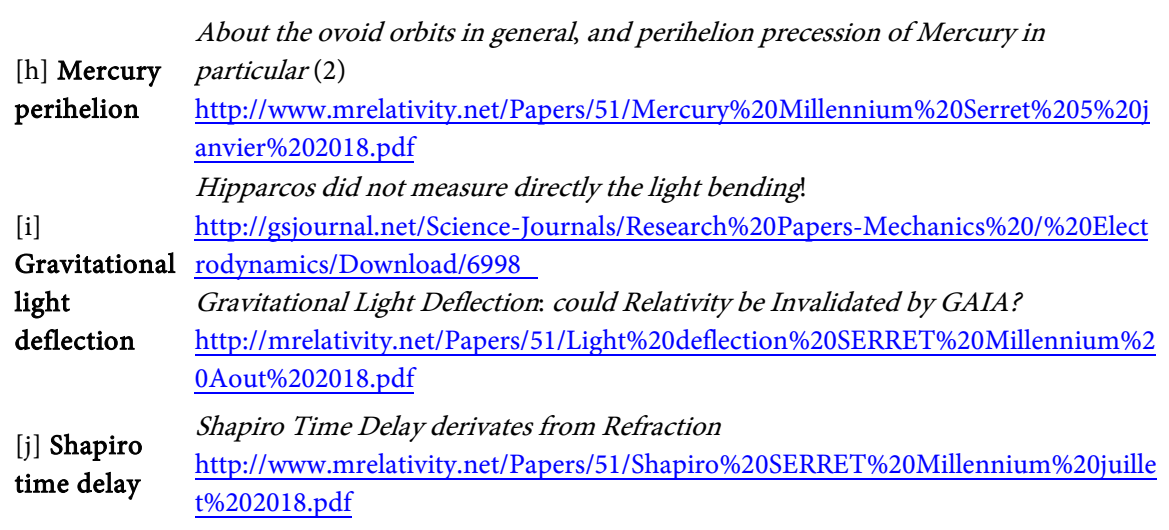

[k] Dispersion The Mass of a Photon estimated from the Pulsar Dispersion Measurement (DM)

Measurement http://gsjournal.net/Science-Journals/Research\%20Papers-Relativity\%20Theory/Do of Pulsars wnload/7490

[1] Pioneer The Pioneer Anomaly explained by the Processing of the Doppler Effect

Anomaly http://gsjournal.net/Science-Journals/Research\%20Papers-Relativity\%20Theory/Do wnload/7330

[m] Dark The flat rotation curve of our galaxy explained within Newtonian mechanics

Matter https://physicsessays.org/browse-journal-2/product/1240-7-olivier-serret-the-flat-ro tation-curve-of-our-galaxy-explained-within-newtonian-mechanics.html

[n] Dark Gravity vs. Dark Energy, about the Expansion of the Universe

Energy http://file.scirp.org/pdf/JMP_2018011714405269.pdf

[o] Gravitational waves or particle radiation?

Gravitational https://www.physicsessays.org/browse-journal-2/product/1588-12-olivier-serret-gra waves vitational-waves-or-particle-radiation.html 
APPENDIX A: G. SAGNAC'S EXPERIMENT

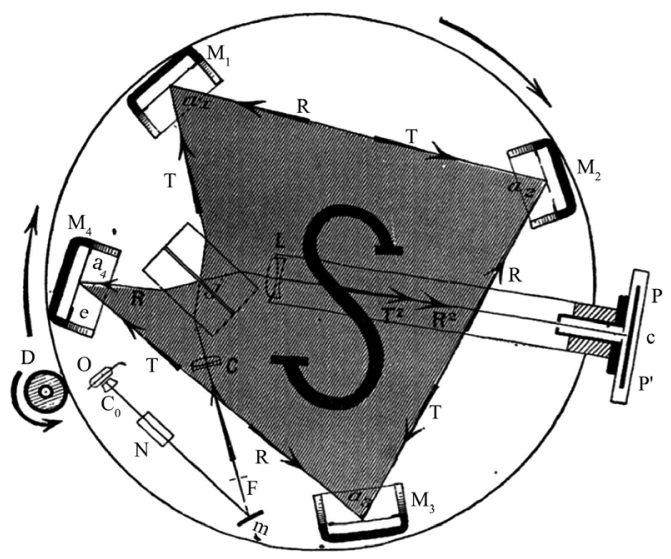

Figure 4. G. Sagnac's experimental setup.

Between two photos of the receiver, one taken with a direction of rotation and the other one in the other direction, Sagnac uses in his experiment (see Figure 4) the parameter $z$ defined as the displacement (relative) of the center of interference:

$$
z=\frac{\Delta c}{\Lambda}[\mathrm{m} / \mathrm{m}]
$$

with

$\Delta c=$ distance between the central fringe $\mathrm{cl}$ in one direction of rotation and the central fringe $\mathrm{c} 2$ in the other direction of rotation.

$\Lambda=$ interfrange $=$ interval between two dark lateral fringes $\mathrm{f}$ (cf middle of paragraph 6 of G. Sagnac's article).

He then obtains as a formula of $z$.

$$
\text { Equation (1) } z=\frac{16 \pi N S}{\lambda c}[\mathrm{~m} / \mathrm{m}]
$$

with

$S$ surface swept by the light beam.

$N$ number of revolutions per second [rps].

Considering the speed of rotation $\Omega$ :

$$
\Omega=2 \pi N[\mathrm{rad} / \mathrm{s}]
$$

we are getting

$$
z=8 \frac{S \Omega}{\lambda c}[\mathrm{~m} / \mathrm{m}]
$$

Note 1:

$z$ is similar to Fringe Shift, except that we usually take the distance between the central fringe $\mathrm{c} 1$ according to a direction of rotation and the central fringe $\mathrm{c} 0$ of the immobile disk, i.e. $\Delta c / 2$.

Thus

$$
F S=\frac{\Delta c / 2}{\Lambda}
$$




$$
\begin{gathered}
F S=\frac{z}{2} \\
\text { Equation (2) } F S=4 \frac{S \Omega}{\lambda c}[\mathrm{~m} / \mathrm{m}]
\end{gathered}
$$

Note 2:

This formula appears coherent, although dissimilar on the sufaces, with that found in the scientific literature [6]:

$$
F S=4 \frac{A \Omega}{\lambda c}[\mathrm{~m} / \mathrm{m}]
$$

with $A$ the Area of the disc.

\section{APPENDIX B: Relativistic Explanations}

Relativity theoriy could explain Sagnac from different ways:

a) By the relativity of the simultaneity [8]:

A simultaneous event in one frame is not in another frame. Two light rays can meet simultaneously in a frame (in motion) and not in another frame (motionless): it is the "temporal" shift $\left({ }^{*}\right)$.

This was put into equation first by Paul Langevin in 1921 [9] [10] [11]:

$$
\left\{\begin{array}{l}
t_{+}=\frac{2 \pi R+\Omega R t_{+}}{c}=\frac{\Omega R t_{+}}{v} \\
t_{-}=\frac{2 \pi R-\Omega R t_{-}}{c}=\frac{\Omega R t_{-}}{v}
\end{array}\right.
$$

b) By time dilatation:

Sagnac effect can be attributed to the difference in the time dilation (or phase change) [12].

c) Length variation

With the rotating disc, the lengths depend on the direction of travel [13].

*: Discussion about the "temporal" shift:

The difficulty of the "temporal" shift explanation is that it is not compatible with the interference of the Young slits $\left.{ }^{* *}\right)$ : the central line corresponds to the same optical path, it moves according to the variation of optical path (and not according to a variation of time, to the relativity of simultaneity or to a "temporal" shift). For the central peak, whatever its movement (at the same light celerity):

$$
t_{+}=t_{-}=t_{i}
$$

and so

$$
\begin{aligned}
& \left\{\begin{array}{l}
c t_{i}=2 \pi R \\
\Omega R t_{i}=0
\end{array}\right. \\
& \left\{\begin{array}{l}
t_{i}=\frac{2 \pi R}{c} ! \\
t_{i}=0 ! !
\end{array}\right.
\end{aligned}
$$


${ }^{*}$ : Principle of the interference peak of Young slits: see Figure 5.

Peak of interference intensity where the 2 paths (at the same light celerity) are equal, where the difference in path is zero.

$$
F S=\frac{\delta L}{\lambda}=\frac{a}{\lambda D} x[\mathrm{~m} / \mathrm{m}]
$$

\section{APPENDIX C: Neo-Newtonian Derivation}

To detail the neo-Newtonian explanation, we will proceed in two steps:

- First we will simplify the experience of G. Sagnac to half a turn.

- Then we will return to the initial case with a complete turn.

First step: simplification of the experimental scheme

The simplification relates to two points:

1) We replace the polygonal surface $S$ by a circular surface.

2) And we change the location of the "opposite" photographic receiver on the disc of the light source (see Figure 6). The distance traveled by the beams being then half less, the offset is then in this assembly half less.

Hypotheses: in (Ro) frame,

- From the geometry of the circle:

$$
A_{0} I^{+}+I^{-} A_{0}=P
$$

with $P$ for Perimeter, or light path.

- Disc in rotation:

$$
\begin{array}{cc}
V=\Omega R \\
& A_{0} A_{1}=\Omega R t_{I} \\
\text { So } \quad B_{0} B_{1}=\Omega R t_{I}
\end{array}
$$

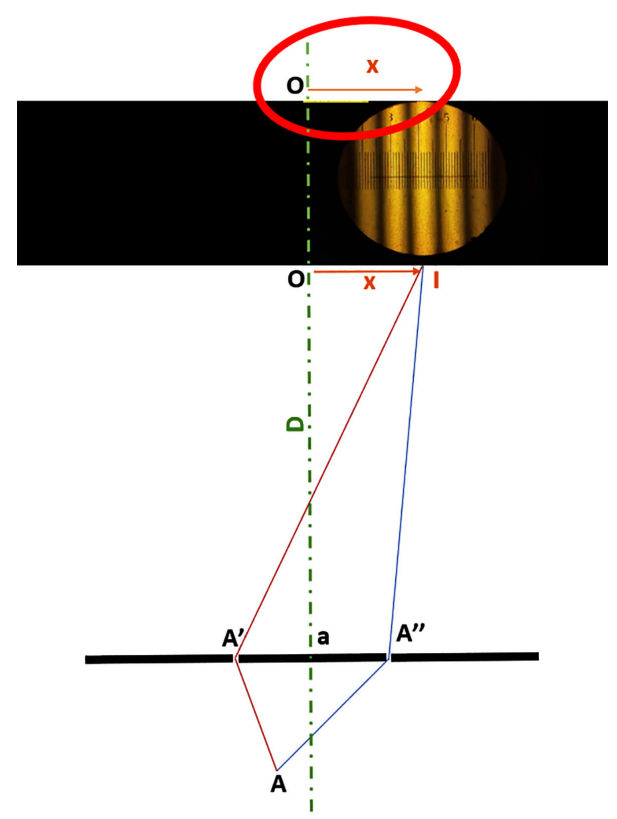

Figure 5. Young interference. 

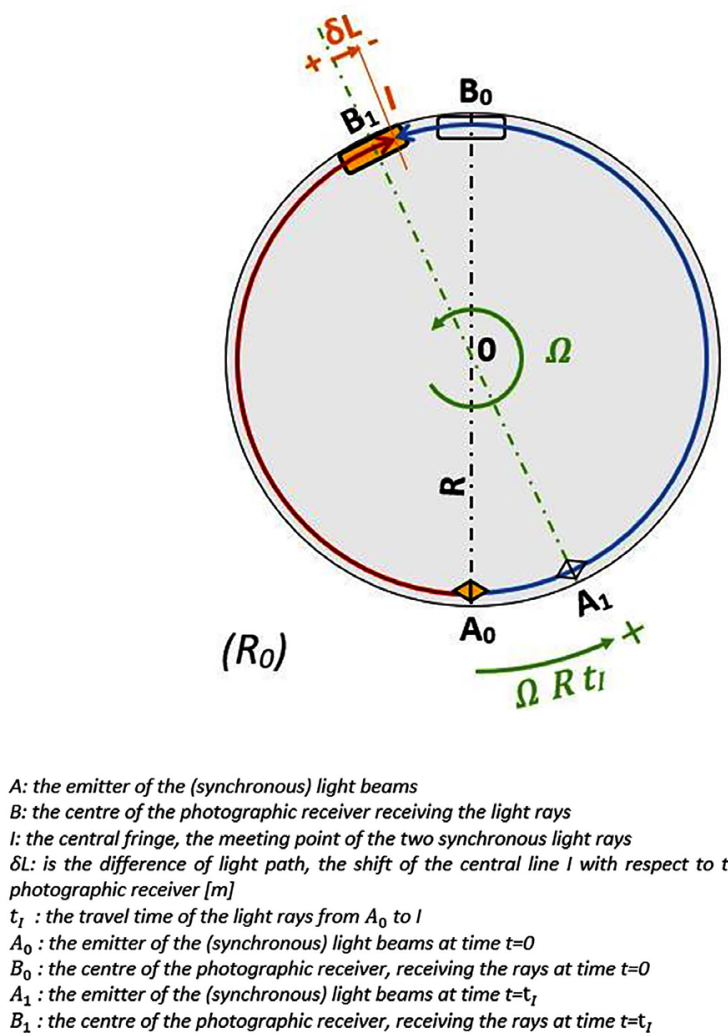

Figure 6. Simplification of the experimental setup.

- Trajectory of the two light rays:

$$
A_{0} I^{+}=c^{+} t_{I}
$$

and

$$
A_{0} I^{-}=-c^{-} t_{I}
$$

as defined

$$
\delta L=B_{1} I
$$

- In Neo-Newtonian Mechanics:

$$
\begin{gathered}
c^{+}=c+\varepsilon \text { with } \varepsilon \cong 0 \\
c^{-}=c-x \cdot V \text { with } 0<x<1
\end{gathered}
$$

Consequence:

From (C7)

$$
\begin{aligned}
& \delta L\left(\frac{1}{2}\right)=B_{1} B_{0}+B_{0} I \\
& \delta L\left(\frac{1}{2}\right)=B_{0} I-B_{0} B_{1}
\end{aligned}
$$

By geometry

$$
\begin{gathered}
A_{0} I=A_{0} B_{0}+B_{0} I \\
\text { So } B_{0} I=A_{0} I-A_{0} B_{0}
\end{gathered}
$$




$$
\text { So } B_{0} I=A_{0} I-P / 2
$$

From (C11), (C14)

$$
\delta L=A_{0} I-P / 2-B_{0} B_{1}
$$

From (C4), (C5)

$$
\delta L\left(\frac{1}{2}\right)=c^{+} t_{I}-\pi R-\Omega R t_{I}
$$

From (C1), (C5), (C6)

$$
\begin{gathered}
c^{+} t_{I}+c^{-} t_{I}=P \\
t_{I}=\frac{P}{c^{+}+c^{-}}
\end{gathered}
$$

From (C8), (C9)

$$
t_{I} \cong \frac{P}{2 c-x \cdot V}
$$

From (C8), (C16), (C19)

$$
\begin{gathered}
\delta L\left(\frac{1}{2}\right)=(c-\Omega R) \frac{P}{2 c-x \cdot V}-P / 2 \\
\delta L\left(\frac{1}{2}\right)=\frac{P c-P \Omega R-c P+P x \cdot V / 2}{2 c-x \cdot V} \\
\delta L\left(\frac{1}{2}\right)=\frac{-P \Omega R+P x \cdot V / 2}{2 c-x \cdot V} \\
V \ll c \delta L\left(\frac{1}{2}\right)=\frac{-P R \Omega+P x \cdot V / 2}{2 c}
\end{gathered}
$$

From (C2)

$$
\begin{gathered}
\delta L\left(\frac{1}{2}\right)=\frac{-P R \Omega+P x \cdot R \Omega / 2}{2 c} \\
\delta L\left(\frac{1}{2}\right)=-\frac{P R \Omega}{2 c}\left(1-\frac{x}{2}\right)
\end{gathered}
$$

Comment: with $x<1$ then $\delta L<0$.

The sign "minus" means that the peak of interference occurs in the vicinity to the right of B1 (cf Figure)

$$
\text { With } x \ll 1 \delta L\left(\frac{1}{2}\right) \approx-\frac{P R \Omega}{2 c}
$$

\section{IV.2 Second step: full rotation}

Second step: Now let's go back to the un-simplified case with a full rotation. In that case you have to double the path and therefore the Equation $(\mathrm{C} 28)$ to:

$$
\text { Equation } 6 \delta L(\text { Neo-Newtonian })=-\frac{P R \Omega}{c}
$$

- Remark 1: in the case of a circle

$$
P R=2 \pi R^{2}=2 A
$$




$$
\delta L(\text { Circle } / \text { Neo-Newtonian })=-2 \frac{A \Omega}{c}
$$

It is half what the theory of Relativity predicts for a circle!

- Remark 2: in the case of a square

$$
\begin{gathered}
P=4 \sqrt{2} R \\
\delta L(\text { Square / Neo-Newtonian })=-4 \frac{\sqrt{2} R^{2} \Omega}{c}
\end{gathered}
$$

